\title{
Autophagy induction enhances tetrandrine-induced apoptosis via the AMPK/mTOR pathway in human bladder cancer cells
}

\author{
BO KOU ${ }^{1 *}$, WEI LIU ${ }^{2 *}, \mathrm{XU} \mathrm{XU}^{3 *}$, YANG YANG $^{1}$, QIUYUE YI $^{1}$, FENGWEI GUO $^{1}$, \\ JIANPENG LI ${ }^{1}$, JINSONG ZHOU ${ }^{4,5}$ and QINGSHAN KOU ${ }^{6}$
}

\begin{abstract}
Departments of ${ }^{1}$ Cadiovascular Sugery, ${ }^{2}$ Urology and ${ }^{3}$ Human Resources, First Affiliated Hospital of the Medical School, Xi'an Jiaotong University; ${ }^{4}$ Department of Human Anatomy, Histology and Embryology, School of Basic Medical Sciences, Xi'an Jiaotong University Health Science Center; ${ }^{5}$ Key Laboratory of Environment and Genes Related to Diseases,

Xi'an Jiaotong University, Chinese Ministry of Education, Xi'an, Shaanxi 710061;

${ }^{6}$ Medical Center, First People's Hospital of Xianyang, Xianyang, Shaanxi 712000, P.R. China
\end{abstract}

Received March 16, 2017; Accepted August 23, 2017

DOI: $10.3892 /$ or.2017.5988

\begin{abstract}
Tetrandrine, a bisbenzylisoquinoline alkaloid isolated from the roots of Stephania tetrandra is a traditional Chinese medicine and exerts anticancer capacity in various types of cancers. Previous studies have shown that tetrandrine induces apoptosis in bladder cancer cells via activation of the caspase cascade. However, the underlying mechanism has not yet been reported. Autophagy is a cellular process involved in the degradation of broken proteins and aging organelles to maintain homeostasis. Recent studies indicate that autophagy is implicated in cancer therapy. Thus, we focused on the correlation between autophagy and apoptosis upon tetrandrine treatment in human bladder cancer cells. Firstly, our results observed a marked increase in autophagic double-membrane vacuoles and fluorescent puncta of red fluorescence proteingreen fluorescence protein-LC3 (GRP-RFP-LC3) upon tetrandrine treatment, as evidenced by transmission electron microscopy and confocal fluorescence microscopy. Secondly, the expression of LC3-II was increased in tetrandrine-treated T24 and 5637 cells in a time- and concentration-dependent manner. Subsequently, downregulation of p62 and LC3 turnover assay further confirmed that tetrandrine induced autophagic flux in bladder cancer T24 and 5637 cells. Thirdly,
\end{abstract}

Correspondence to: Professor Jinsong Zhou, Department of Human Anatomy, Histology and Embryology, School of Basic Medical Sciences, Xi'an Jiaotong University Health Science Center, 76 West Yanta Road, Xi'an, Shaanxi 710061, P.R. China

E-mail: zjs301@mail.xjtu.edu.cn

Dr Qingshan Kou, Medical Center, First People's Hospital of Xianyang, Xianyang, Shaanxi 712000, P.R. China

E-mail: 492526094@qq.com

${ }^{*}$ Contributed equally

Key words: tetrandrine, bladder cancer, autophagy, apoptosis, AMPK, mTOR the protein levels of phosphorylated-AMP-activated protein kinase (AMPK) and phosphorylated-acetyl-coenzyme A carboxylase (ACC) were upregulated in the tetrandrine-treated cells, while the mammalian target of rapamycin (mTOR)related proteins were downregulated. Moreover, AICAR, a common AMPK activator, further increased the expression the LC3-II, while AMPK inhibitor compound C partially reversed the LC3-II protein levels in bladder cancer T24 cells. Finally, AICAR significantly reinforced the growth inhibition and apoptosis induction of tetrandrine in T24 and 5637 cells, while compound $\mathrm{C}$ had an opposite effect, suggesting that AMPK-mediated autophagy enhanced the cytotoxic and pro-apoptosis effect of tetrandrine in human bladder cancer cells. Taken together, the present study showed that tetrandrine induced autophagy in human bladder cancer cells by regulating the AMPK/mTOR signaling pathway, which contributed to the apoptosis induction by tetrandrine, indicating that tetrandrine may be a potential anticancer candidate for the treatment of bladder cancer, and autophagy may be a possible mechanism for cancer therapy.

\section{Introduction}

Bladder cancer is one of the most common malignancies of the urogenital system worldwide with an estimated 300,00 new cases emerging every year (1). Non-muscle-invasive bladder and muscle-invasive bladder cancer are the main two patterns of bladder cancer (2). Most early-stage bladder cancer is recognized as non-muscle invasive bladder cancer, which is effectively treated by surgery. However, due to the high recurrence of bladder cancer after surgical resection, most advanced bladder cancer develops into a muscle-invasive pattern resulting in lethal disease without effective treatment (3). Thus, novel potential agents are urgently needed for the treatment of bladder cancer, and Chinese medicine has attracted great attention for cancer therapeutics.

Tetrandrine, a bisbenzylisoquinoline alkaloid, is isolated from the roots of Stephania tetrandra (4). Numerous studies have been reported that tetrandrine can be broadly used for patients with arthritis, hypertension, sepsis, inflammation 
and silicosis (5-9). Additionally, tetrandrine has been found to inhibit proliferation and induce apoptosis in various types of cancer cells, such as gastric and colon cancer, hepatocellular and renal cell carcinoma (10-13). Moreover, previous research has shown that tetrandrine induced cell cycle arrest in neuroblastoma cells (14). In addition, tetrandrine was reported to have the capacity to reverse multidrug resistance in cancer cells, indicating the strong anticancer activity of tetrandrine (15). However, there is little research concerning the effect of tetrandrine on bladder cancer. Only one study showed that apoptosis induction by tetrandrine was mediated via activation of the caspase cascade in bladder cancer T24 and 5637 cells, but the underlying mechanism is still undefined (16).

In recent years, autophagy has gained considerable attention in regards to tumor progression, particularly in cell apoptosis (17). Autophagy is a conserved cellular process for the degradation of broken proteins and aging organelles to maintain intercellular homeostasis (18). Recent research demonstrated that autophagy may be a potential antitumor mechanism. It was revealed that inhibition of autophagy facilitated epithelial-mesenchymal transition by reactive oxygen species (ROS)/HO-1 signaling pathway in human ovarian cancer cells (19).

In our previous study, we confirmed that tetrandrine at low concentrations reversed epithelial-mesenchymal transition in bladder cancer cells (20). Nevertheless, whether tetrandrine could have the ability to induce autophagy in bladder cancer cells is unknown. In the present study, we revealed that autophagy induction by tetrandrine enhanced tetrandrine-induced apoptosis in bladder cancer cells, which was mediated by the AMPK/mTOR signaling pathway. Our results demonstrated that tetrandrine may be a potential anticancer agent, while autophagy may be a crucial mechanism for cancer therapy.

\section{Materials and methods}

Reagents and cell culture. Tetrandrine $\left(\mathrm{C}_{38} \mathrm{H}_{42} \mathrm{~N}_{2} \mathrm{O}_{6}\right)$ was purchased from Sigma-Aldrich (St. Louis, MO, USA) and stock solutions with a concentration of $25 \mathrm{mg} / \mathrm{ml}$ were stored at $-20^{\circ} \mathrm{C}$. Antibodies against microtubule-associated protein 1 light chain 3- $\beta$ (LC3-I), LC3-II, p62, p-AMPK (Thr172), AMPK, p-ACC (Ser79), ACC, p-mTOR, mTOR, p70S6K (Thr389), p70S6K (Ser371) and p-4EBP1 (Thr37/46) were purchased from Cell Signaling Technology (Beverly, MA, USA). Mouse monoclonal $\beta$-actin antibody was purchased from CWBIO (Beijing, China). Bafilomycin A1 (Baf A1), AICAR, compound $\mathrm{C}$ (Com C) and 3-(4,5-dimethylthiazol-2-yl)-2,5-diphenyltetrazolium bromide (MTT) were obtained from Sigma Chemical Co. (St. Louis, MO, USA). GFP-RFP-LC3 adenovirus was purchased from Hanbio (Shanghai, China). The enhanced chemiluminescence (ECL) detection system was obtained from Amersham Life Science, Inc. (Arlington Heights, IL, USA).

Human bladder cancer T24 and 5637 cell lines were obtained from the American Type Culture Collection (ATCC; Manassas, VA, USA) and maintained in Dulbecco's modified Eagle's medium, supplemented with $10 \%$ fetal bovine serum and $1 \%$ penicillin-streptomycin (Invitrogen, Carlsbad, CA,
USA). Cells were incubated in a humidified atmosphere with $5 \% \mathrm{CO}_{2}$ at $37^{\circ} \mathrm{C}$.

Transmission electron microscopy. After treatment, cells were washed with phosphate-buffered saline (PBS) and fixed with glutaraldehyde ( $\mathrm{pH}$ 7.4). Then, the cells were treated with $1 \%$ osmium tetroxide and dehydrated. Subsequently, the cells were embedded in Ultracut (Leica Ultracut R; Leica Microsystems, Bensheim, Germany) and cut into 60-nm sections, followed by uranyl acetate and lead citrate staining. Ultimately, the ultrathin sections were visualized under an Hitachi H07650 transmission electron microscope (Hitachi, Ltd., Tokyo, Japan).

Confocal fluorescence microscopy. T24 cells were cultured on slides and transfected with GFP-RFP-LC3 adenovirus. After treatment with tetrandrine, cells were washed with PBS and fixed with $4 \%$ paraformaldehyde. Then, the slides were blocked with glycerol, and the localization of LC3 puncta was observed with the confocal fluorescence microscope (Nikon, Tokyo, Japan).

Western blotting. Briefly, the treated cells were washed with PBS buffer and lysed with RIPA buffer [10 $\mathrm{mmol} / 1$ Tris- $\mathrm{HCl}$ (pH 7.4), $150 \mathrm{mmol} / \mathrm{l} \mathrm{NaCl}, 0.1 \%$ sodium dodecyl sulfate (SDS), $1 \mathrm{mmol} / 1$ ethylenediaminetetraacetic acid, $1 \mathrm{mmol} / \mathrm{l}$ ethylene glycol tetraacetic acid, $0.3 \mathrm{mmol} / \mathrm{l}$ phenylmethylsulfonyl fluoride, $0.2 \mathrm{mmol} / 1$ sodium orthovanadate, $1 \% \mathrm{NP}-40,10 \mathrm{mg} / \mathrm{ml}$ leupeptin and $10 \mathrm{mg} / \mathrm{ml}$ aprotinin], containing proteinase and phosphatase inhibitors on ice. After centrifugation and denaturation, $30 \mu \mathrm{g}$ of clarified cell lysate was separated by SDS-polyacrylamide gel (10 or 15\%) and transferred to polyvinylidene fluoride membranes. Then, the membranes were blocked with $5 \%$ non-fat milk for $1 \mathrm{~h}$ at room temperature and incubated with primary antibody at $4^{\circ} \mathrm{C}$ overnight. After being washed with Tris-buffered saline with Tween-20 (TBST) buffer, the bands were then incubated with horseradish peroxidase-conjugated secondary antibody for $1 \mathrm{~h}$. Ultimately, the protein bands were analyzed using an ECL detection system, followed by exposure to X-ray film.

MTT assay. Briefly, $6 \times 10^{3}$ cells were seeded onto 96-well plates and treated with different treatments for $24 \mathrm{~h}$. Then, $20 \mu \mathrm{l}$ of MTT dye solution was mixed with $180 \mu \mathrm{l}$ of medium to each well and incubated at $37^{\circ} \mathrm{C}$ for $4 \mathrm{~h}$. Subsequently, cells were lysed with dimethyl sulfoxide was used to dissolve the formazan crystals. Finally, the optical density (OD) of each well was detected at a wavelength of $570 \mathrm{~nm}$ using a 96-well microplate reader (Bio-Rad, Hercules, CA, USA).

Flow cytometry. The bladder cancer T24 and 5637 cells were exposed to a certain treatment for $24 \mathrm{~h}$. The treated cells were washed, collected and stained with fluorescein isothiocyanate (FITC)-conjugated Annexin V and propidium iodide (PI) according to the manufacturer's protocol. The apoptotic cells were subsequently analyzed by flow cytometry (BD FACScan flow cytometer; BD Biosciences, San Diego, CA, USA). The experiments were performed in triplicate.

Statistical analysis. The results are presented as the mean \pm SD. All statistical analyses were performed using 
A
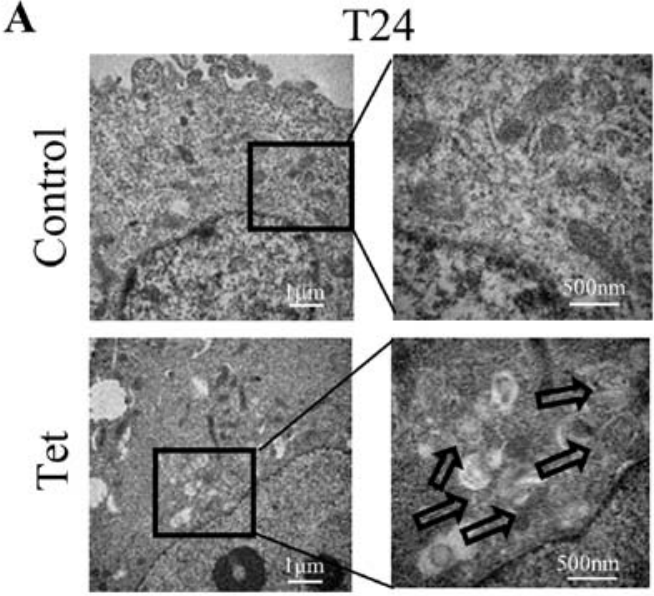

C
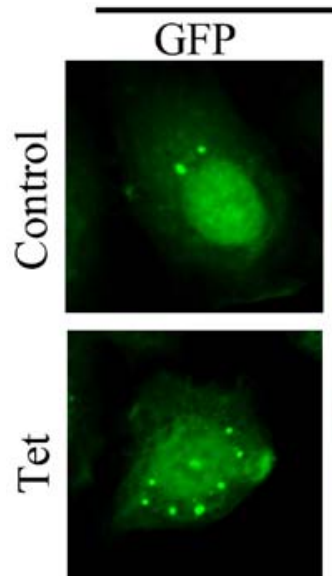

T24
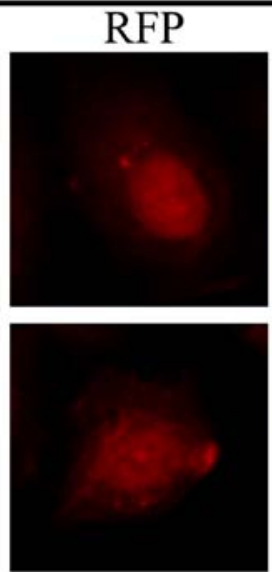

B
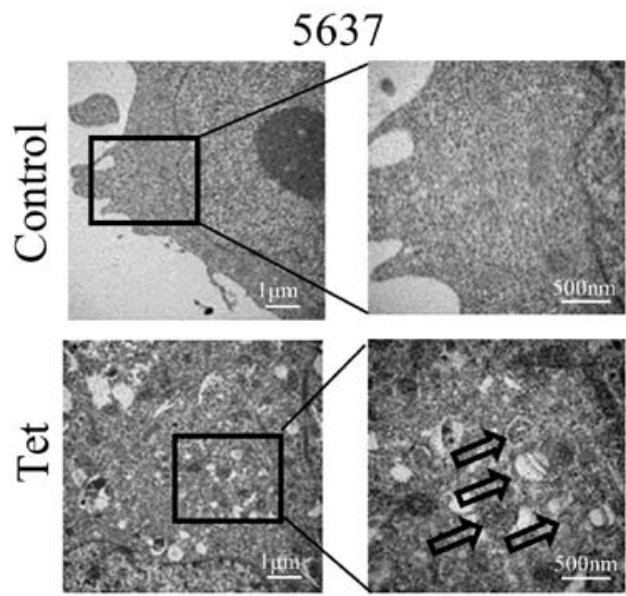

D

T24
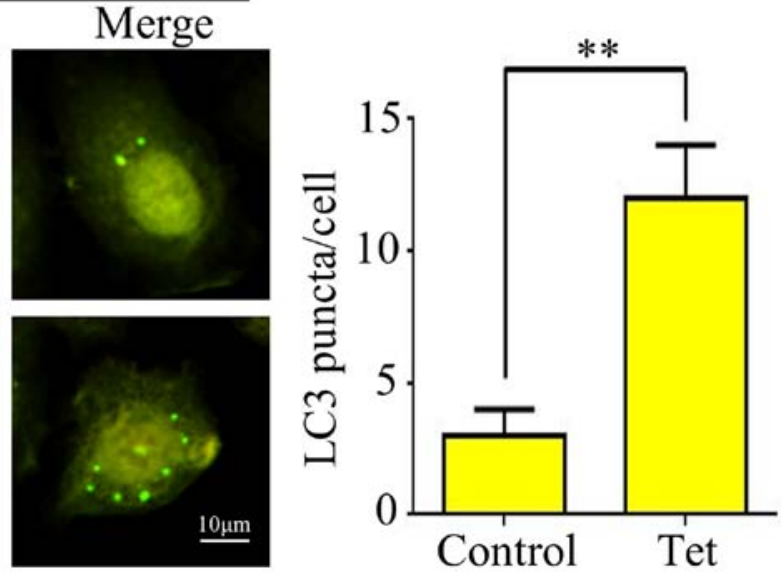

Figure 1. Tetrandrine induces autophagic vacuoles in bladder cancer T24 and 5637 cell lines. The representative double-membrane structure of autophagic vesicles is shown in (A) T24 and (B) 5637 cells treated with tetrandrine (Tet) $(10 \mu \mathrm{M})$ for 24 h using transmission electron microscopy. The arrows indicate autophagic vesicles. Scale bars, $1 \mu \mathrm{m}$ (left), $400 \mathrm{~nm}$ (right). (C) Representative fluorescence photomicrographs and (D) quantification of GFP-RFP-LC3 puncta in T24 cells stably transfected with LC3 adenovirus and treated with Tet $(10 \mu \mathrm{M})$ for 24 h. Yellow puncta denote autophagic vesicle structures. Scale bar, $10 \mu \mathrm{m}$. Results represents mean \pm SD of 3 independent experiments; ${ }^{* *} \mathrm{P}<0.01$, statistically significant.

GraphPad Prism 5.2 software. Student's t-test (two-sided) was used for comparisons involving only two groups. $\mathrm{P}<0.05$ was identified as a significant difference.

\section{Results}

Tetrandrine induces autophagic vacuoles in bladder cancer T24 and 5637 cell lines. Since there is no study concerning the correlation between tetrandrine and autophagy in bladder cancer, we firstly treated T24 (Fig. 1A) and 5637 (Fig. 1B) cell lines with $10 \mu \mathrm{M}$ tetrandrine and observed the ultrastructure of the cells by transmission electron microscopy. A significant increase in autophagic double-membrane vacuoles was observed in the tetrandrine-treated cells compared with this number in the control group.

The conversion of LC3-I to LC3-II is a hallmark of mammalian autophagy. Thus, we transfected the GFP-RFP-LC3 adenovirus containing acid-sensitive GFP and the acid-insensitive RFP into bladder cancer T24 cells to explore the effect of tetrandrine on autophagy. Microscopic examination showed a marked increase in fluorescent puncta of GRP-RFP-LC3, indicating that tetrandrine induced autophagosome formation and the occurrence of autophagy (Fig. 1C and D).
Tetrandrine induces autophagic flux in bladder cancer T24 and 5637 cells. To further verify whether tetrandrine induces autophagy in bladder cancer cells, the levels of LC3-II, a membrane bound form of LC3, were evaluated by western blotting. Results are presented in Fig. $2 \mathrm{~A}$ and $\mathrm{C}$. Expression of LC3-II in T24 cells was gradually increased in a time- and concentration-dependent manner following tetrandrine treatment. Similar results were found in the 5637 cells (Fig. 2B and D). p62, an indicator of lysosome degradation, has been used for monitoring autophagic flux (21). Our findings showed a marked decline in the p62 level upon tetrandrine treatment in a time- and concentration-dependent pattern (Fig. 2A-D).

To further validate the induction of autophagic flux by tetrandrine, T24 and 5637 cells were exposed to the combination treatment of tetrandrine and Baf A1, an inhibitor of lysosomal acidification. Obviously, the LC3-II levels were increased in both the control and tetrandrine-treated cells in the presence of Baf A1. Moreover, the combined group exhibited a higher level of LC3-II compared with that in the tetrandrine alone or Baf A1 alone treated groups (Fig. 2E and F). These results suggested that an increase in production, not a decrease in degradation, resulted in the accumulation of LC3-II by 


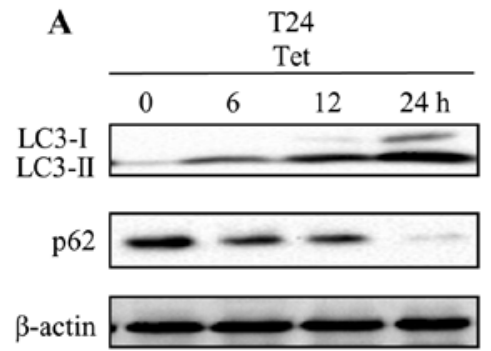

B
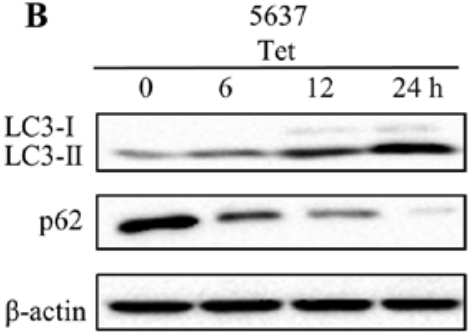

C

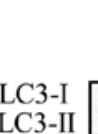

$$
\begin{array}{r}
\mathrm{p} 62 \\
\beta \text {-actin }
\end{array}
$$

D

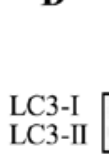

p62

$\beta$-actin

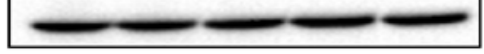

$\mathrm{T} 24$

Tet

\begin{tabular}{lllll}
0 & 1.25 & 2.5 & $5.0 \quad 10.0 \mu \mathrm{M}$ \\
\hline
\end{tabular}

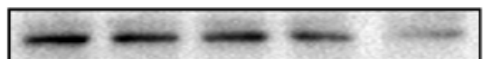

5637

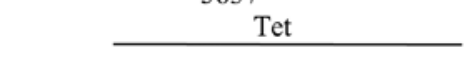

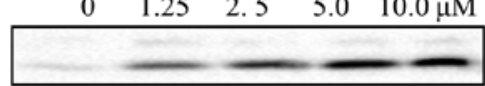

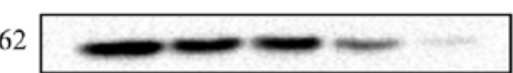

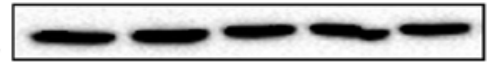

$\mathbf{E}$

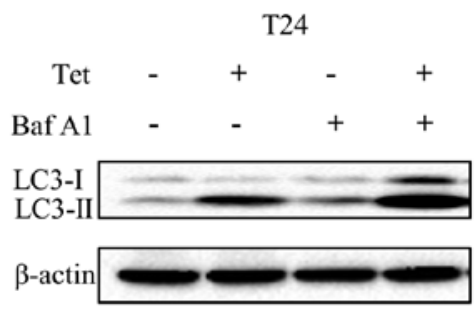

$\mathbf{F}$

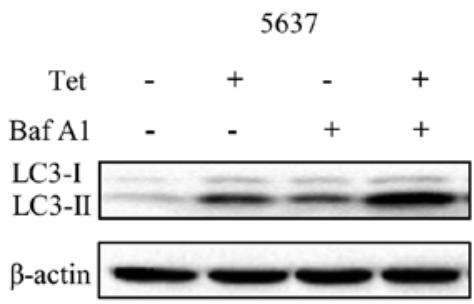

Figure 2. Tetrandrine induces autophagic flux in bladder cancer T24 and 5637 cells. Western blot detection of LC3-I, LC3-II, p62 and $\beta$-actin in (A and C) T24 and (B and D) 5637 cells treated with tetrandrine (Tet) for various times $(0,6,12$ and $24 \mathrm{~h})$ and concentrations $(0,1.25,2.5,5$ and $10 \mu \mathrm{M})$. Western blot detection of LC3-I, LC3-II and $\beta$-actin in (E) T24 and (F) 5637 cells treated with Tet in the presence or absence of Baf A1 (10 nM).

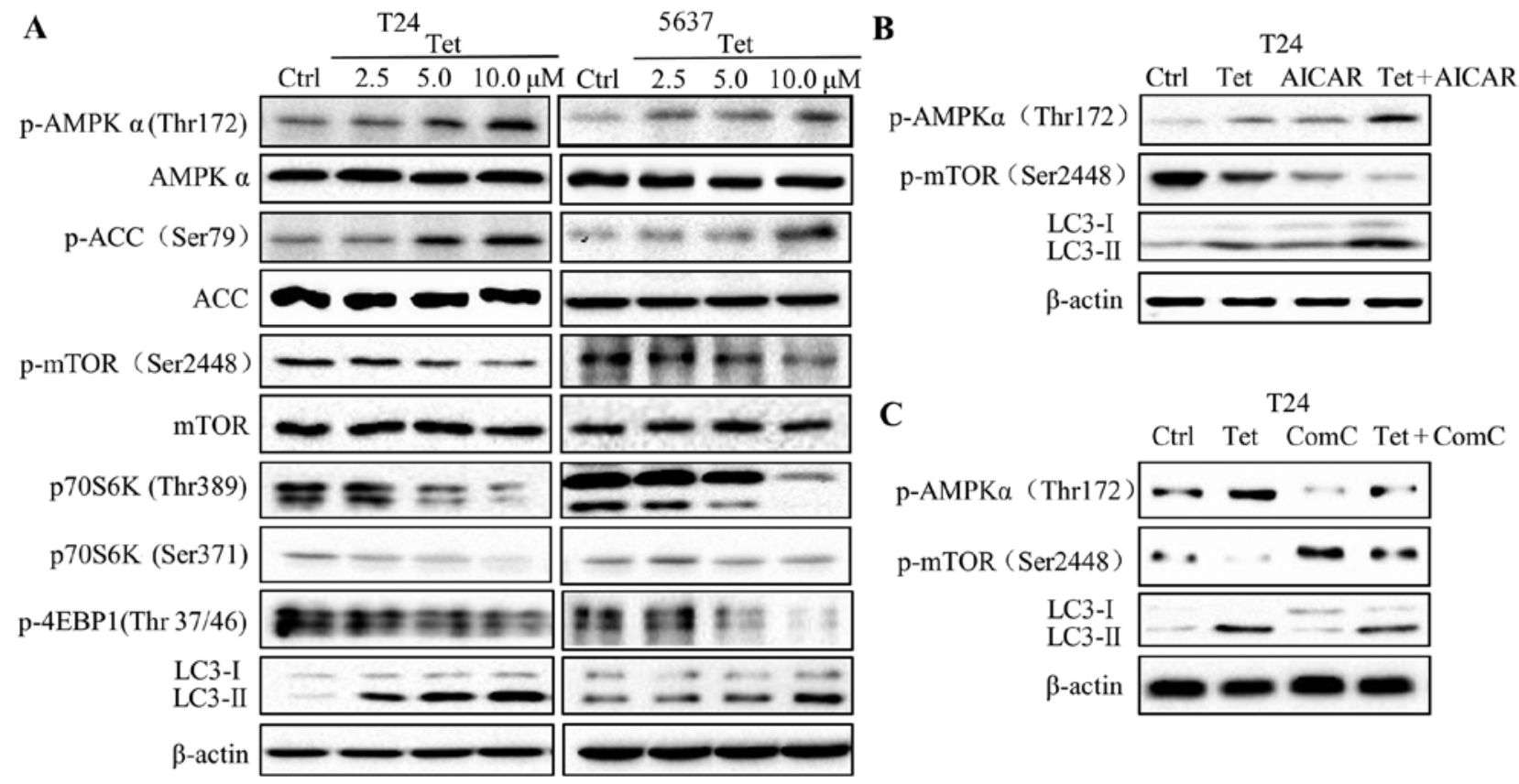

Figure 3. Tetrandrine induces autophagy through the AMPK/mTOR pathway. (A) Western blot detection of p-AMPK (Thr172), AMPK, p-ACC (Ser79), ACC, p-mTOR (Ser2448), mTOR, p70S6K (Thr389), p70S6K (Ser371), p-4EBP1 (Thr 37/46), LC3-I, LC3-II and $\beta$-actin in T24 and 5637 cells treated with tetrandrine (Tet) for $24 \mathrm{~h}$. Western blot detection of p-AMPK (Thr172), p-mTOR (Ser2448), LC3-I, LC3-II and $\beta$-actin in T24 cells treated with Tet accompanied by AMPK inhibitor compound C (Com C; $10 \mu \mathrm{M})(\mathrm{B})$ and activator AICAR $(1 \mathrm{mM})(\mathrm{C})$ for $24 \mathrm{~h}$.

tetrandrine, and that tetrandrine significantly induced autophagic flux in the bladder cancer cells.

Tetrandrine induces autophagy through the AMPK/mTOR pathway. AMPK is a critical energy sensor that regulates energy metabolism in eukaryotic cells (22). Recent studies report that autophagy can be induced by AMP-activated protein kinase (AMPK). To determine the role of AMPK in tetrandrine-induced autophagy, we evaluated the protein levels of phosphorylated- and total-AMPK after tetrandrine treatment. The findings showed that the levels of phosphorylated-AMPK
(Thr172) were markedly increased in a dose-dependent manner in the T24 and 5637 cells following tetrandrine treatment, but no significant changes were presented in totalAMPK levels (Fig. 3A). Consistent with the above results, the protein level of phosphorylated-ACC (Ser79), a substrate of phosphorylated-AMPK, is upregulated by tetrandrine. mTOR, a negative regulator in the autophagy process, has been verified to be regulated by AMPK. To examine the role of mTOR signaling in tetrandrine-induced autophagy, we detected the phosphorylation levels of mTOR, and subsequently observed a decline in p-mTOR (Ser2448) (Fig. 3A). In accordance with 

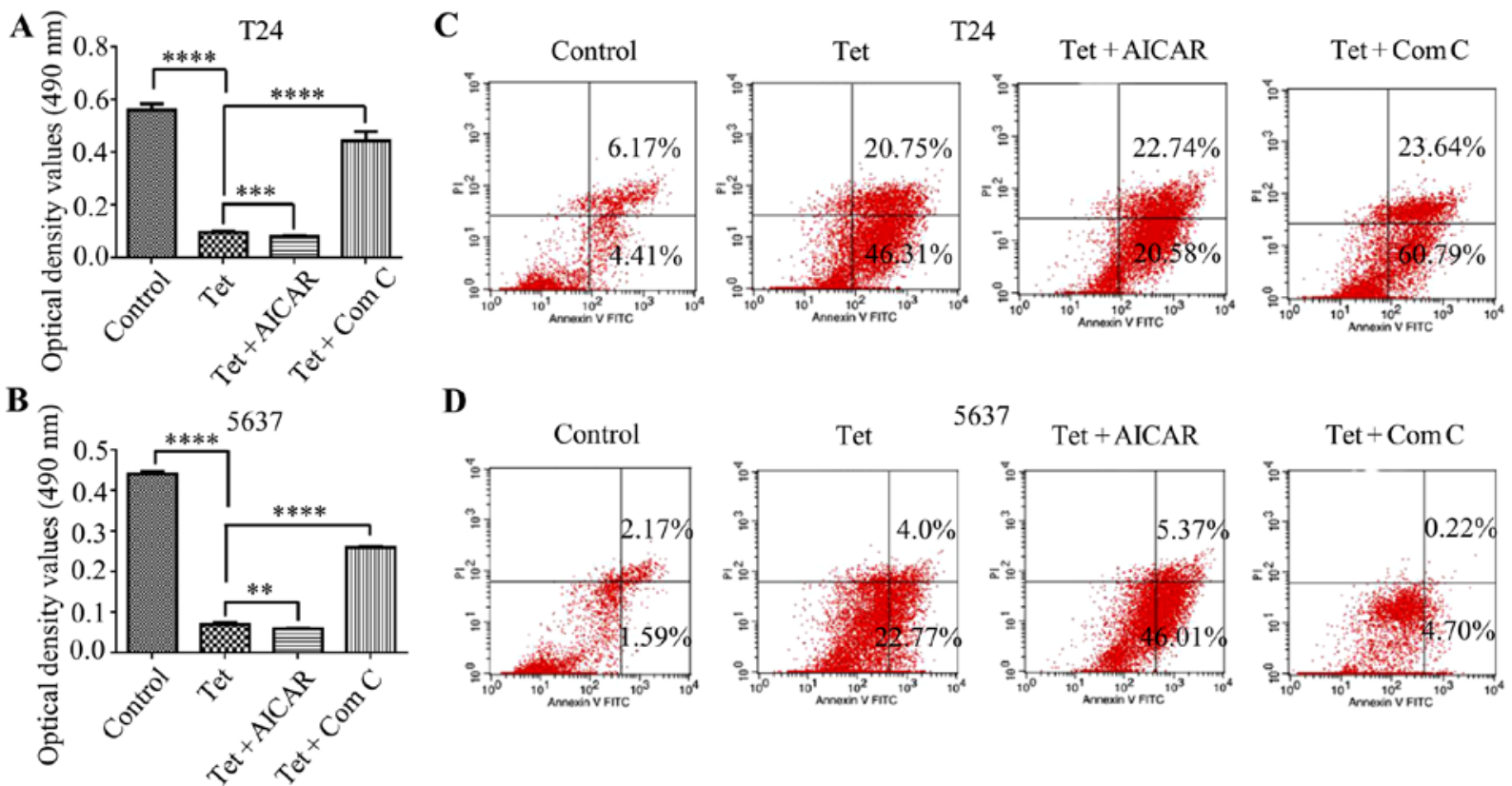

D
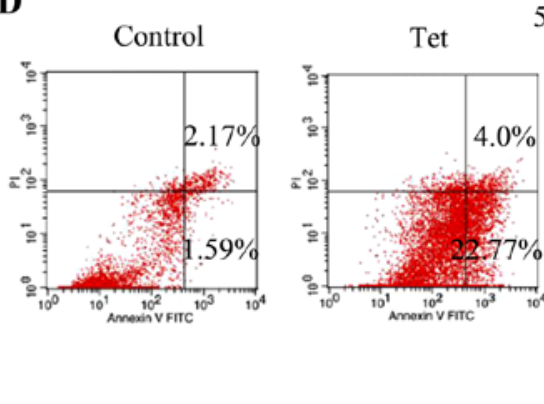

5637
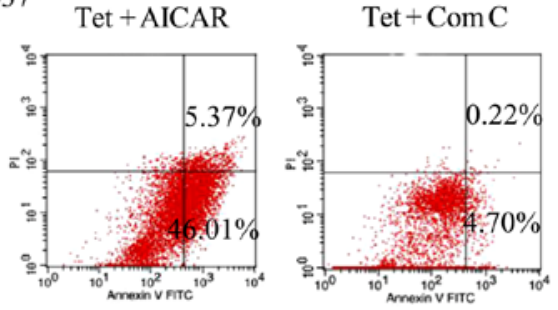

Figure 4. Autophagy induction enhances the cytotoxic effect of tetrandrine in bladder cancer T24 and 5637 cells. (A) T24 and (B) 5637 cells were synergistically treated with tetrandrine (Tet) and AICAR or Com C for $24 \mathrm{~h}$. The optical density values $(490 \mathrm{~nm})$ were measured by MTT assay. Results represents mean \pm SD of 3 independent experiments ${ }^{* * *} \mathrm{P}<0.01,{ }^{* * *} \mathrm{P}<0.001$ and ${ }^{* * * *} \mathrm{P}<0.0001$, statistically significant. Flow cytometric analysis of the apoptotic effect of different treatmentc (control, Tet, Tet + AICAR, Tet + Com C) on (C) T24 and (D) 5637 cells. After treatment for $24 \mathrm{~h}$, cells were collected and stained with Annexin V/PI followed by FACS analysis.

the above results, the phosphorylation levels of eukaryotic initiation factor 4E-binding protein (4E-BP1) and p70S6K, two downstream substrates of mTOR, were also downregulated in the tetrandrine-treated T24 and 5637 cells (Fig. 3A).

To further confirm the role of AMPK in tetrandrine-induced autophagy, AICAR (AMPK activator) and compound C (AMPK inhibitor) were used for combination treatment with tetrandrine. The results showed that AICAR further increased the LC3-II levels and decreased the p-mTOR levels compared with tetrandrine alone in the T24 cells (Fig. 3B). Reversely, compound $\mathrm{C}(\mathrm{Com} \mathrm{C})$ inhibited the high expression of LC3-II, while restored the low expression of p-mTOR, suggesting that tetrandrine induced autophagy by regulating the AMPK/ mTOR signaling pathway (Fig. 3C).

Autophagy induction enhances the cytotoxic effect of tetrandrine in bladder cancer T24 and 5637 cells. The activation of autophagy has been shown to be correlated with apoptosis induction. To address whether AMPK-mediated autophagy contributes to tetrandrine-induced autophagy, T24 and 5637 cells were treated with tetrandrine and AICAR to explore the change of tetrandrine on the cytotoxic effect. As expected, AICAR further enhanced the cytotoxic effect of tetrandrine in the T24 (Fig. 4A) and 5637 (Fig. 4B) cells. On the contrary, compound $\mathrm{C}$ partially reversed the growth inhibition of tetrandrine in these two bladder cancer cell lines (Fig. 4A and B). Consistent with the above results, the subsequent analysis of flow cytometry showed that AICAR reinforced the apoptosis induction of tetrandrine in the T24 (Fig. 4C) and 5637 (Fig. 4D) cells, while compound $\mathrm{C}$ had the opposite effect (Fig. 4C and D). Therefore, these results indicated that AMPK-mediated autophagy contributed to tetrandrineinduced apoptosis in bladder cancer cells.

\section{Discussion}

Numerous studies have demonstrated that tetrandrine has promising capacity in cancer therapy, but the effect of tetrandrine on human bladder cancer remains unknown. It has been reported that tetrandrine induces apoptosis by triggering the caspase cascade in bladder cancer T24 and 5637 cells (16). Yet, the underlying mechanism of apoptosis induction by tetrandrine is still unclear. Accumulating evidence indicates that autophagy is a dynamic cellular process that involves the lysosomal degradation of broken proteins and aging organelles to ensure cellular survival. In addition, autophagy has been implicated in cancer progression. It was demonstrated that tetrandrine induced autophagy in human hepatocellular carcinoma via mitochondrial dysfunction, ROS accumulation and activation of the extracellular signal-regulated kinase (ERK) signaling pathway (23). In addition, tetrandrine induced autophagy by triggering ROS generation and upregulating Notch1 signaling in acute premyelocytic leukemia cells (24). In contrast, tetrandrine was identified as a potent lysosomal deacidification agent to block autophagic flux in prostate cancer PC-3 and renal cell carcinoma 786-O cells (25). However, the correlation between tetrandrine and autophagy in human bladder cancer cells has not yet been confirmed. In the present study, tetrandrine induced autophagic vacuoles in bladder cancer cells, as evidenced by an increase in autophagic double-membrane structure and fluorescent LC3 puncta. Moreover, analysis of LC3-II and p62 levels, and the subsequent LC3 turnover assay further confirmed that autophagic flux could be induced by tetrandrine treatment.

Various signaling pathways have been implicated in the regulation of autophagy. Research has shown that cobalt chloride induced autophagy and apoptosis in glioma cells by the 
p53 pathway (26). Another study revealed that isomahanineinduced autophagy was mediated by p38 mitogen-activated protein kinase (MAPK) signaling pathway in oral squamous cell carcinoma cells (27). Among the various pathways, the AMPK pathway has attracted great attention. AMPK is a vital kinase in autophagy regulation, which senses cellular energy status to maintain intercellular homeostasis (28). Research supports that AMPK plays a crucial role in autophagy induction in response to various stresses, such as starvation (29). In the present study, tetrandrine markedly increased p-AMPK and p-ACC in the T24 and 5637 cells. mTOR, a downstream substrate of AMPK, negatively regulates autophagy. Research as also demonstrated that hypoxia promotes autophagy in nucleus pulposus cells independent of mTOR signaling (30). In the present study, the phosphorylated levels of mTOR, 4E-BP1 and p70S6K were downregulated upon tetrandrine treatment. Then, the combined treatment of tetrandrine and AICAR or compound $\mathrm{C}$ in the subsequent assay suggested that tetrandrine-induced autophagy was mediated by the AMPK/ mTOR signaling pathway.

It has been widely reported that autophagy is a double-edged sword in tumor development and suppression (31). In response to adverse stress, such as nutrient starvation, autophagy may be triggered for the degradation of unnecessary molecules, serving as a potential survival mechanism to maintain intercellular homeostasis, regulate the immune response and remodel development $(32,33)$. Studies suggest that autophagy induction is a mechanism of chemoresistance $(34,35)$. On the contrary, numerous anticancer agents, such as resveratrol, induce autophagic cell death, indicating that autophagy may be a vital mechanism for anticancer therapy (36). Results showed that 3-methyladenine (3-MA) and bafilomycin A1 partially restored the antiproliferative effect of tetrandrine on human oral cancer cells (37). In the present study, the AMPK activator AICAR reinforced the growth inhibition and apoptosis induction of tetrandrine in T24 and 5637 cells, while compound C protected tetrandrine-treated bladder cancer cells against a decrease in cell viability, indicating that AMPK-mediated autophagy contributed to tetrandrine-induced apoptosis in human bladder cancer cells.

In conclusion, our findings indicate that activation of AMPK signaling is critical for tetrandrine-induced autophagy in bladder cancer cells, which enhances the apoptosis induction of tetrandrine. Tetrandrine may be an alternative anticancer candidate for the treatment of bladder cancer, and autophagy may be a possible mechanism for cancer therapy.

\section{Acknowledgements}

The present study was partially supported by the National Natural Science Foundation of China (no. 81602562), the Fundamental Research Funds for the Central University of Xi'an Jiaotong University (no. 1191329722), and the Institutional Scientific Development Foundation of the First Affiliated Hospital of Xi'an Jiaotong University (no. 2015YK17).

\section{References}

1. Siegel RL, Miller KD and Jemal A: Cancer statistics, 2016. CA Cancer J Clin 66: 7-30, 2016.
2. Panebianco V, De Berardinis E, Barchetti G, Simone G, Leonardo C, Grompone MD, Del Monte M, Carano D, Gallucci M, Catto J, et al: An evaluation of morphological and functional multi-parametric MRI sequences in classifying non-muscle and muscle invasive bladder cancer. Eur Radiol 27: 3759-3766, 2017.

3. Witjes JA, Compérat E, Cowan NC, De Santis M, Gakis G, Lebret T, Ribal MJ, Van der Heijden AG and Sherif A; European Association of Urology: EAU guidelines on muscle-invasive and metastatic bladder cancer: Summary of the 2013 guidelines. Eur Urol 65: 778-792, 2014.

4. Liu T, Liu X and Li W: Tetrandrine, a Chinese plant-derived alkaloid, is a potential candidate for cancer chemotherapy. Oncotarget 7: 40800-40815, 2016.

5. Yuan X, Tong B, Dou Y, Wu X, Wei Z and Dai Y: Tetrandrine ameliorates collagen-induced arthritis in mice by restoring the balance between Th17 and Treg cells via the aryl hydrocarbon receptor. Biochem Pharmacol 101: 87-99, 2016.

6. Zhang J, Yu B, Zhang XQ, Sheng ZF, Li SJ, Wang ZJ, Cui XY, Cui SY and Zhang YH: Tetrandrine, an antihypertensive alkaloid, improves the sleep state of spontaneously hypertensive rats (SHRs). J Ethnopharmacol 151: 729-732, 2014.

7. Ye Z, Van Dyke K and Rossan RN: Effective treatment with a tetrandrine/chloroquine combination for chloroquine-resistant falciparum malaria in Aotus monkeys. Malar J 12: 117, 2013.

8. Kang OH, An HJ, Kim SB, Mun SH, Seo YS, Joung DK, Choi JG, Shin DW and Kwon DY: Tetrandrine suppresses proinflammatory mediators in PMA plus A23187-induced HMC-1 cells. Int J Mol Med 33: 1335-1340, 2014.

9. Fu NF, Luo CH, Wu JC, Zheng YY, Gan YJ, Ling JA, Liang HQ, Liang DY, Xie J, Chen XQ, et al: Clearance of free silica in rat lungs by spraying with chinese herbal kombucha. Evid Based Complement Alternat Med 2013: 790792, 2013.

10. Li X, Lu X, Xu H, Zhu Z, Yin H, Qian X, Li R, Jiang X and Liu B: Paclitaxel/tetrandrine coloaded nanoparticles effectively promote the apoptosis of gastric cancer cells based on 'oxidation therapy'. Mol Pharm 9: 222-229, 2012.

11. Wu JM, Chen Y, Chen JC, Lin TY and Tseng SH: Tetrandrine induces apoptosis and growth suppression of colon cancer cells in mice. Cancer Lett 287: 187-195, 2010.

12. Liu C, Gong K, Mao X and Li W: Tetrandrine induces apoptosis by activating reactive oxygen species and repressing Akt activity in human hepatocellular carcinoma. Int J Cancer 129: 1519-1531, 2011.

13. Chen S, Liu W, Wang K, Fan Y, Chen J, Ma J, Wang X, He D, Zeng $\mathrm{J}$ and $\mathrm{Li} \mathrm{L}$ : Tetrandrine inhibits migration and invasion of human renal cell carcinoma by regulating Akt/NF- $\kappa \mathrm{B} / \mathrm{MMP}-9$ signaling. PLoS One 12: e0173725, 2017.

14. Jin Q, Kang C, Soh Y, Sohn NW, Lee J, Cho YH, Baik HH and Kang I: Tetrandrine cytotoxicity and its dual effect on oxidative stress-induced apoptosis through modulating cellular redox states in Neuro 2a mouse neuroblastoma cells. Life Sci 71: 2053-2066, 2002.

15. Lu Y, Li F, Xu T and Sun J: Tetrandrine prevents multidrug resistance in the osteosarcoma cell line, U-2OS, by preventing Pgp overexpression through the inhibition of NF- $\mathrm{kB}$ signaling. Int J Mol Med 39: 993-1000, 2017.

16. Li X, Su B, Liu R, Wu D and He D: Tetrandrine induces apoptosis and triggers caspase cascade in human bladder cancer cells. J Surg Res 166: e45-e51, 2011.

17. Ravegnini G, Sammarini G, Nannini M, Pantaleo MA, Biasco G, Hrelia P and Angelini S: Gastrointestinal stromal tumors (GIST): Facing cell death between autophagy and apoptosis. Autophagy 13: 452-463, 2017.

18. Galluzzi L, Pietrocola F, Levine B and Kroemer G: Metabolic control of autophagy. Cell 159: 1263-1276, 2014.

19. Zhao Z, Zhao J, Xue J, Zhao X and Liu P: Autophagy inhibition promotes epithelial-mesenchymal transition through ROS/HO-1 pathway in ovarian cancer cells. Am J Cancer Res 6: 2162-2177, 2016.

20. Zhang Y, Liu W, He W, Zhang Y, Deng X, Ma Y, Zeng J and Kou B: Tetrandrine reverses epithelial-mesenchymal transition in bladder cancer by downregulating Gli-1. Int J Oncol 48: 2035-2042, 2016.

21. Hewitt G, Carroll B, Sarallah R, Correia-Melo C, Ogrodnik M, Nelson G, Otten EG, Manni D, Antrobus R, Morgan BA, et al: SQSTM1/p62 mediates crosstalk between autophagy and the UPS in DNA repair. Autophagy 12: 1917-1930, 2016.

22. Carling D: AMPK signalling in health and disease. Curr Opin Cell Biol 45: 31-37, 2017. 
23. Gong K, Chen C, Zhan Y, Chen Y, Huang Z and Li W: Autophagyrelated gene 7 (ATG7) and reactive oxygen species/extracellular signal-regulated kinase regulate tetrandrine-induced autophagy in human hepatocellular carcinoma. J Biol Chem 287: 35576-35588, 2012.

24. Liu T, Men Q, Wu G, Yu C, Huang Z, Liu X and Li W: Tetrandrine induces autophagy and differentiation by activating ROS and Notch1 signaling in leukemia cells. Oncotarget 6: 7992-8006, 2015.

25. Qiu W, Su M, Xie F, Ai J, Ren Y, Zhang J, Guan R, He W, Gong Y and Guo Y: Tetrandrine blocks autophagic flux and induces apoptosis via energetic impairment in cancer cells. Cell Death Dis 5: e1123, 2014

26. Cheng BC, Chen JT, Yang ST, Chio CC, Liu SH and Chen RM: Cobalt chloride treatment induces autophagic apoptosis in human glioma cells via a p53-dependent pathway. Int J Oncol 50: 964-974, 2017.

27. Utaipan T, Athipornchai A, Suksamrarn A, Chunsrivirot S and Chunglok W: Isomahanine induces endoplasmic reticulum stress and simultaneously triggers p38 MAPK-mediated apoptosis and autophagy in multidrug-resistant human oral squamous cell carcinoma cells. Oncol Rep 37: 1243-1252, 2017.

28. Hardie DG: AMP-activated/SNF1 protein kinases: Conserved guardians of cellular energy. Nat Rev Mol Cell Biol 8: 774-785, 2007.

29. Vingtdeux V, Giliberto L, Zhao H, Chandakkar P, Wu Q, Simon JE, Janle EM, Lobo J, Ferruzzi MG, Davies P, et al AMP-activated protein kinase signaling activation by resveratrol modulates amyloid-beta peptide metabolism. J Biol Chem 285: 9100-9113, 2010
30. Choi H, Merceron C, Mangiavini L, Seifert EL, Schipani E, Shapiro IM and Risbud MV: Hypoxia promotes noncanonical autophagy in nucleus pulposus cells independent of MTOR and HIF1A signaling. Autophagy 12: 1631-1646, 2016.

31. White E, Mehnert JM and Chan CS: Autophagy, metabolism, and cancer. Clin Cancer Res 21: 5037-5046, 2015.

32. Cheng Y, Ren X, Hait WN and Yang JM: Therapeutic targeting of autophagy in disease: Biology and pharmacology. Pharmacol Rev 65: 1162-1197, 2013.

33. Höhn A and Grune T: Lipofuscin: Formation, effects and role of macroautophagy. Redox Biol 1: 140-144, 2013.

34. Kim M, Jung JY, Choi S, Lee H, Morales LD, Koh JT, Kim SH, Choi YD, Choi C, Slaga TJ, et al: GFRA1 promotes cisplatin-induced chemoresistance in osteosarcoma by inducing autophagy. Autophagy 13: 149-168, 2017.

35. Jin F, Wang Y, Li M, Zhu Y, Liang H, Wang C, Wang F, Zhang CY, Zen K and Li L: MiR-26 enhances chemosensitivity and promotes apoptosis of hepatocellular carcinoma cells through inhibiting autophagy. Cell Death Dis 8: e2540, 2017.

36. Chang CH, Lee CY, Lu CC, Tsai FJ, Hsu YM, Tsao JW, Juan YN, Chiu HY, Yang JS and Wang CC: Resveratrol-induced autophagy and apoptosis in cisplatin-resistant human oral cancer CAR cells: A key role of AMPK and Akt/mTOR signaling. Int J Oncol 50: 873-882, 2017.

37. Huang AC, Lien JC, Lin MW, Yang JS, Wu PP, Chang SJ and Lai TY: Tetrandrine induces cell death in SAS human oral cancer cells through caspase activation-dependent apoptosis and LC3-I and LC3-II activation-dependent autophagy. Int J Oncol 43: 485-494, 2013. 